\title{
A Framework for Modeling Agent-Oriented Software*
}

\author{
Haiping Xu and Sol M. Shatz \\ Department of Electrical Engineering and Computer Science \\ The University of Illinois at Chicago \\ Chicago, IL 60607 \\ Email: \{hxul,shatz\}@eecs.uic.edu
}

\begin{abstract}
With the increasing importance of complex software systems in the software industry, the need for using agent technologies to develop large-scale commercial and industrial software systems is growing rapidly. Such systems are complex and there is a pressing need for system modeling techniques to support reliable, maintainable and extensible design. G-Nets are a type of Petri net defined to support modeling of a system as a set of independent and loosely-coupled modules. In this paper, we first introduce an extension of G-Nets, agent-based $G$ Net, as a generic model for agent design. Then to progress from an agent-based design model to an agent-oriented model, new mechanisms to support inheritance modeling are introduced. To illustrate our formal modeling technique for multi-agent systems, an example of an agent family in electronic commerce is provided.
\end{abstract}

\section{Introduction}

With the increasing importance of complex software systems in the software industry, the need for using agent technologies to develop large-scale commercial and industrial software systems is growing rapidly. Technologies for multi-agent systems (MAS) stem from distributed artificial intelligence (DAI) research [1], and MAS are usually defined as concurrent systems based on the notion of autonomous, reactive, internally-motivated agents in a decentralized environment [2]. The increasing interest in MAS research is due to the significant advantages inherent in such systems, including their ability to solve problems that may be too large for a centralized single agent, to provide enhanced speed and reliability, and to tolerate uncertain data and knowledge [1].

Although there are many efforts on developing multiagent systems, there is a lack of research on formal specification and design of such systems [3][4]. As multiagent technology begins to emerge as a viable solution for

\footnotetext{
* This material is based upon work supported by the U.S. Army Research Office under grant number DAAD19-99-1-0350, and the NSF under grant number CCR-9988168.
}

large-scale industrial and commercial applications, there is an increasing need to ensure that the systems being developed are robust, reliable and fit for purpose [4]. Previous work on formal modeling multi-agent systems includes: (1) using formal languages, such as $Z$, to provide a framework for describing a system at different levels of abstractions; (2) using temporal modal logic to allow the dynamic aspects of agents; (3) designing formal languages, such as DESIRE, for multi-agent specification [4][12]. Unlike previous work, our approach uses the principle of "separation of concerns" in agent-oriented design. We separate the traditional object-oriented features and reasoning mechanisms in our agent-oriented software model as much as possible, and we discuss how reuse can be achieved in agent-oriented design.

In this paper, we extend a formal model, called a G-Net (a form of Petri net [7]), to support inheritance modeling of agent classes in multi-agent systems. The advantage of our formal mechanism is that it provides a clean interface between agents with asynchronous communication ability and supports formal reasoning for an agent design. Furthermore, our formal mechanism is based on Petri net formalism, which is a mature formal model in terms of both existing theory and tool support.

\section{An Agent-based Model}

\subsection{The Standard G-Net Model}

A widely accepted software engineering principle is that a system should be composed of a set of independent modules, where each module hides the internal details of its processing activities and modules communicate through well-defined interfaces. The G-Net model provides strong support for this principle [8]. G-Nets are an object-based extension of Petri nets. We assume that the reader has a basic understanding of Petri nets [7], so we begin with some introduction to the G-Net model. A G-Net system is composed of a number of G-Nets, each of them representing a self-contained module or object. A G-Net is composed of two parts: a special place called Generic Switch Place (GSP) and an Internal Structure (IS). The GSP provides the abstraction of the module, and serves as the only interface between the G-Net and other modules. 
The $I S$, a modified Petri net, represents the detailed design of the module. An example of G-Nets is shown in Figure 1. Here the G-Net models represent two objects - the Buyer and the Seller. The generic switch places are represented by $G S P($ Buyer $)$ and $G S P($ Seller $)$ enclosed by ellipses, and the internal structures of these models are represented by round-cornered rectangles that contain the detailed design of four methods: buyGoods(), askPrice(), returnPrice() and sellGoods(). In G.IS, the internal structure of G-Net $G$, Petri net places represent primitives, while transitions, together with arcs, represent connections or relations among those primitives. The primitives may define local actions or method calls. Method calls are represented by special places called Instantiated Switch Places (ISP). A primitive becomes enabled if it receives a token, and an enabled primitive can be executed. Given a G-Net $G$, an ISP of $G$ is a 2-tuple $\left(G^{\prime} . N i d, m t d\right)$, where $G$ ' could be the same G-Net $G$ or some other G-Net, Nid is a unique identifier of G-Net $G^{\prime}$, and $m t d$ is a method defined in $G^{\prime} . I S$. Each $I S P\left(G^{\prime} . N i d, m t d\right)$ denotes a method call $m t d\left(\right.$ ) to G-Net $G^{\prime}$. An example ISP (denoted as an ellipsis in Figure 1) is shown in the method askPrice() defined in G-Net Buyer, where the method askPrice() makes a method call returnPrice() to the G-Net Seller to query about the price for some goods.

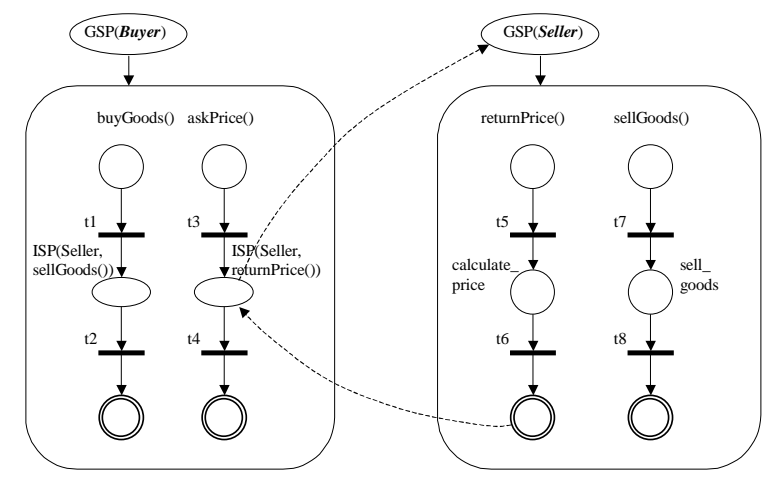

Figure 1. G-Net model of buyer and seller objects

From the above description, we can see that a G-Net model essentially represents a module or an object rather than an abstraction of a set of similar objects. In a recent paper [9], we have extended the G-Net model to support class modeling. The idea of this extension is to generate a unique object identifier, G.Oid, and initialize the state variables when a G-Net object is instantiated from a G-Net $G$. An ISP method invocation is no longer represented as the 2-tuple $\left(G^{\prime}\right.$. Nid, $\left.m t d\right)$, instead it is the 2-tuple (G'.Oid, $m t d)$, where different object identifiers could be associated with the same G-Net class model.

The token movement in a G-Net object is similar to that of original G-Nets [8]. A token $t k n$ is a triple (seq, sc, $m t d$ ), where $s e q$ is the propagation sequence of the token, $s c$ is the status color of the token and $m t d$ is a triple ( $m t d \_n a m e$, para_list, result). The usage of these elements can be found in [8][9]. For ordinary places, tokens are removed from input places and deposited into output places by firing transitions. However, for the special ISP places, the output transitions do not fire in the usual way. For example, in Figure 1, when the Buyer object calls the returnPrice() method of the Seller object, the token in place $I S P($ Seller, returnPrice ()$)$ is removed and a token is deposited into the GSP place GSP(Seller). Through the $G S P$ of the called G-Net object Seller, the token is then dispatched into an entry place of the appropriate called method, i.e., returnPrice(), for the token contains the information to identify the called method. During "execution" of the method, the token will reach a return place (denoted by double circles) with the result attached to the token. As soon as this happens, the token will return to the ISP of the caller. At this time, the output transition (i.e., $t 4$ in Figure 1) can become enabled and fire.

We call a G-Net model that supports class modeling a standard G-Net model. Notice that the example we provide in Figure 1 follows the Client-Server paradigm, in which a Seller object works as a server and a Buyer object is a client. Although the standard G-Net model works well in object-based design, it is not sufficient in agent-based design for the following reasons. First, agents in multiagent systems are usually developed by different vendors independently, and those agents will be widely distributed across large-scale networks such as the Internet. To make it possible for those agents to communicate with each other, it is essential for them to have a common communication language and to follow common protocols. However the standard G-Net model does not directly support protocol-based language communication between agents. Second, the underlying agent communication model is usually asynchronous, and an agent may decide whether to perform actions requested by some other agents. The standard G-Net model does not directly support asynchronous message passing and decisionmaking, but only supports synchronous method invocations in the form of ISP places. Third, agents are commonly designed to determine their behavior based on individual goals and their knowledge. They may autonomously and spontaneously initiate internal or external behavior at any time. Standard G-Net models can only directly support a predefined flow of control.

\subsection{Extending G-Nets to Support Agent Modeling}

To support agent-based design, we first need to extend a G-Net to support modeling an agent class. The basic idea is similar to extending a G-Net to support class modeling for object-based design [9]. When we instantiate an agentbased G-Net (an agent class model) $G$, an agent identifier G.Aid is generated and the mental state of the resulting agent object (an active object [4]) is initialized. In addition, at the class level, five special modules are introduced to make an agent autonomous and internallymotivated, namely the Goal module, the Plan module, the 
Knowledge-base module, the Environment module and the Planner module. The outline of an agent-based G-Net model is shown in Figure 2. We describe each of the additional modules as follows. A Goal module is an abstraction of a goal model [5], which describes the goals that an agent may possibly adopt. A Plan module is an abstraction of a plan model [5] that consists of a set of plans. A plan may be intended or committed, and only committed plans will be achieved. A Knowledge-base module is an abstraction of a belief model [5], which describes the information about the environment and internal state that an agent of that class may hold. An Environment module is an abstract model of the environment, i.e., the model of the outside world that of interest to the agent and that can be sensed by the agent. The Planner module can be viewed as the heart of an agent, where committed plans are achieved. It may decide to ignore an incoming message, to start a new conversation, or to continue with the current conversation The Goal, Plan and Knowledge-base modules of an agent are updated after each communicative act or if the environment changes.

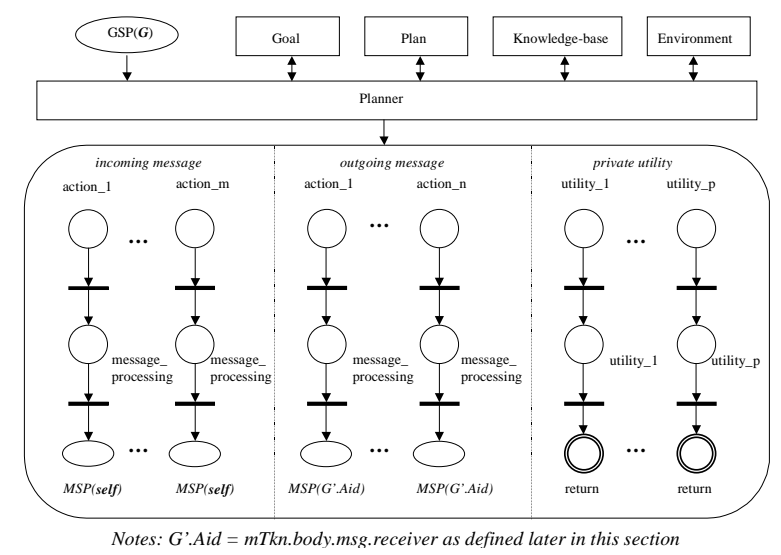

Figure 2. A generic agent-based G-Net model

The internal structure (IS) of an agent model consists of three sections: incoming message, outgoing message, and private utility. The incoming/outgoing message section defines a set of message processing units (MPU), which correspond to a subset of communicative acts [10][11]. Each $M P U$, labeled as action_ $i$ in Figure 2, is used to process incoming/outgoing messages, and may use ISPtype modeling for calls to methods defined in its private utility section. Only the agent itself can call those private utility functions defined in its private utility section.

Although both objects (passive objects) and agents (agent objects) use message-passing to communicate with each other, message-passing for objects is a unique form of method invocation, while agents distinguish different types of messages and model these messages frequently as speech-acts and use complex protocols to negotiate [4]. In particular, these messages must satisfy standardized communicative (speech) acts that define the type and the content of the message (e.g., the FIPA agent communication language, or KQML) [10][11]. Note that in Figure 2, each named MPU action_ $i$ refers to a communicative act, thus our agent-based model supports an agent communication interface. In addition, agents analyze these messages and can decide whether to execute the requested action. As we stated before, agent communications are typically based on asynchronous message passing. Since asynchronous message passing is more fundamental than synchronous message passing, it is useful for us to introduce a new mechanism, called Message-passing Switch Place (MSP), to directly support asynchronous message passing. When a token reaches an $M S P$ (we represent it as an ellipsis in Figure 2), the token is removed and deposited into the GSP of the called agent. But, unlike with the standard G-Net ISP mechanism, the calling agent does not wait for the token to return before it can continue to execute its next step. Since we usually do not think of agents as invoking methods of one-another, but rather as requesting actions to be performed [12], in our agent-based model, we restrict the usage of ISP mechanisms, so they are only used to refer to an agent itself. Thus, in our models, all communications between agents must be carried out through asynchronous message passing as provided by the MSP mechanism.

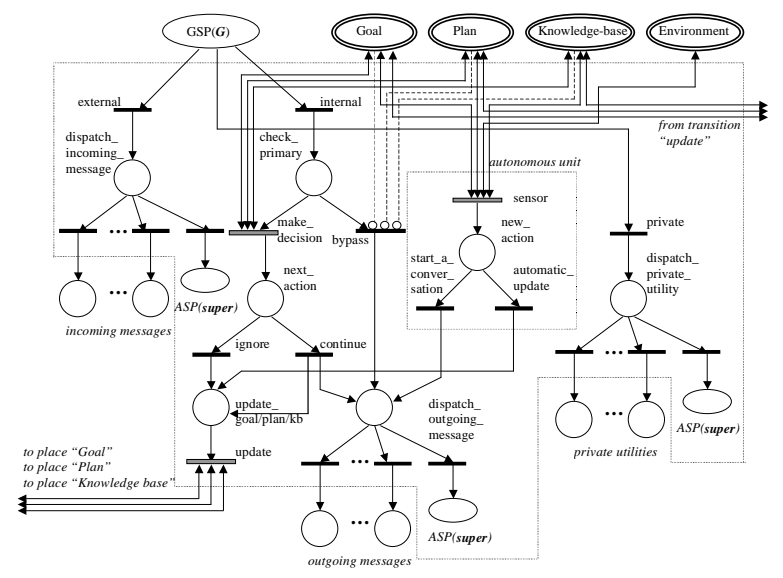

Figure 3. A template of planner module

A template of the Planner module is shown in Figure 3. The modules Goal, Plan, Knowledge-base and Environment are represented as four special places (denoted by double ellipses in Figure 3), each of which contains a token that represents a set of goals, a set of plans, a set of beliefs and a model of the environment, respectively. These four modules connect with the Planner module through abstract transitions, denoted by shaded rectangles in Figure 3 (e.g., the abstract transition make_decision). Abstract transitions represent abstract units of decision-making or mental-state-updating. At a more detailed level of design, abstract transitions would be 
refined into sub-nets; however how to make decisions and how to update an agent's mental state is beyond the scope of this paper, and will be considered in our future work. In the Planner module, there is a unit called autonomous unit that makes an agent autonomous and internally-motivated. An autonomous unit contains a sensor (represented as an abstract transition), which may fire whenever the preconditions of some committed plan are satisfied or when new events are captured from the environment. If the abstract transition sensor fires, based on an agent's current mental state (goal, plan and knowledge-base), the autonomous unit will then decide whether to start a conversation or to simply update its mental state. This is done by firing either the transition start_a_conversation or the transition automatic_update after executing any necessary actions associated with place new_action.

Note that the Planner module is both goal-driven and event-driven because the transition sensor may fire when any committed plan is ready to be achieved or any new event happens. In addition, the Planner module is also message-triggered because certain actions may initiate whenever a message arrives (either from some other agent or from the agent itself). A message is represented as a message token with a tag of internal/external/private. A message token with a tag of external represents an incoming message which comes from some other agent, or a newly generated outgoing message before sending to some other agent; while a message token with a tag of internal is a message forwarded by an agent to itself with the MSP mechanism. In either case, the message token with the tag of internal/external should not be involved in an invocation of a method call. On the contrary, a message token with a tag of private indicates that the token is currently involved in an invocation of some method call. When an incoming message/method arrives, with a tag of external/private in its corresponding token, it will be dispatched to the appropriate MPU/method defined in the internal structure of the agent. If it is a method invocation, the method defined in the private utility section of the internal structure will be executed, and after the execution, the token will return to the calling unit, i.e., an ISP of the calling agent. However, if it is an incoming message, the message will be first processed by a $M P U$ defined in the incoming message section in the internal structure of the agent. Then the tag of the token will be changed from external to internal before it is transferred back to the $G S P$ of the receiver agent by using $\operatorname{MSP}($ self $)$. Here the keyword self refers to the agent object itself. Upon the arrival of a token tagged as internal in a GSP, the transition internal may fire, followed by the firing of the abstract transition make_decision. Note that at this point of time, there would exist tokens in those special places Goal, Plan and Knowledge-base, so the transition bypass is disabled (due to the "inhibitor arc") and may not fire (the purpose of the transition bypass is for inheritance modeling, which will be addressed in Section 2.3). Any necessary actions may be executed in place next_action before the conversation is either ignored or continued. If the current conversation is ignored, the transition ignore fires; otherwise, the transition continue fires. If the transition continue fires, a newly constructed outgoing message, in the form of a token with a tag of external, will be dispatched into the appropriate $M P U$ in the outgoing message section of the internal structure of the agent. After the message is processed by the $M P U$, the message will be sent to a receiver agent by using the $M S P$ (Receiver) mechanism. In either case, a token will be deposited into place update_goal/plan/ $\mathrm{kb}$, allowing the abstract transition update to fire. As a consequence, the Goal, Plan and Knowledge-base modules are updated if needed, and the agent's mental state may change.

As a result of this extension, the structure of tokens in the agent-based G-Net model should be redefined. Essentially there are five types of tokens, namely the message token $m T k n$, the goal token $g T k n$, the plan token $p T k n$, the knowledge token $k T k n$ and the environment token $e T k n$. One way to construct the $g T k n, p T k n, k T k n$ and $e T k n$ is as linked lists. In other words, a $g T k n$ represents a list of goals, $p T k n$ represents a list of plans, a $k T k n$ represents a list of facts, and an $e T k n$ represents a list of events that are of the agent's interests. Since these four types of tokens confine themselves to those special places of their corresponding modules, we do not describe them further in this paper.

A $m T k n$ is a 2-tuple (tag, body), where tag $\in$ \{internal, external, private $\}$ and body is a variant, which is determined by the tag. According to the tag, the token deposited in a GSP will finally be dispatched into a $M P U$ or a method defined in the internal structure of the agentbased G-Net. Then the body of the token $m T k n$ will be interpreted differently. More specifically, we define the $m T k n$ body as follows:

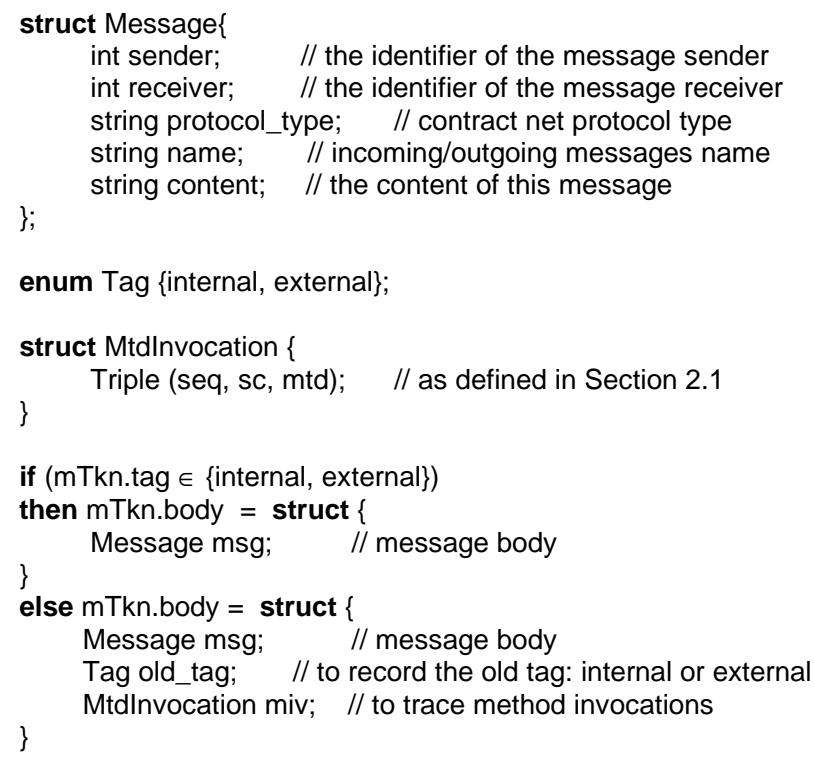


When mTkn.tag $\in$ internal, external $\}$, and an ISP method call occurs, the following steps will take place:

1. The two variables old_tag and miv are attached to the token $m T k n$ to define mTkn.body.old_tag and mTkn.body.miv, respectively. Then, mTkn.tag (the current tag, either internal or external) is recorded into $m T$ Tn.body.old_tag, and mTkn.tag is temporarily set to private.

2. Further method calls are traced by the variable $m T k n . b o d y . m i v$, which is a triple of (seq, $s c, m t d$ ). The tracing algorithm is as defined in [8].

3. After all the ISP method calls are finished and the $m T k n$ token returns to the original ISP, the $m T$ Tkn.tag is set back as mTkn.body.old_tag, and both the variables old_tag and miv are detached.

We now provide a few key definitions giving the formal structure of our agent-based G-Net models.

Definition 2.1 An agent-based $G$-Net is a 7-tuple $A G=$ $(G S P, G L, P L, K B, E N, P N, I S)$, where GSP is a Generic Switch Place providing an abstract for $A G, G L$ is a Goal module, $P L$ is a Plan module, $K B$ is a Knowledge-base module, $E N$ is an Environment module, $P N$ is a Planner module, and $I S$ is an internal structure of $A G$.

Definition 2.2 A Planner module of an agent-based G-Net $A G$ is a colored sub-net defined as a 7-tuple (IGS, IGO, $I P L, I K B, I E N, I I S, D M U)$, where IGS, IGO,IPL, IKB, $I E N$ and IIS are interfaces with GSP, Goal module, Plan module, Knowledge-base module, Environment module and internal structure of $A G$, respectively. $D M U$ is a set of decision-making unit, and it contains three abstract transitions: make_decision, sensor and update.

Definition 2.3 An internal structure (IS) of an agent-based G-Net $A G$ is a triple (IM, OM, $P U)$, where $I M / O M$ is the incoming/outgoing message section, which defines a set of message processing units (MPU); and $P U$ is the private utility section, which defines a set of methods.

Definition 2.4 A message processing unit (MPU) is a triple $(P, T, A)$, where $P$ is a set of places consisting of three special places: entry place, ISP and MSP. Each MPU has only one entry place and one $M S P$, but it may contain multiple ISPs. $T$ is a set of transitions, and each transition can be associated with a set of guards. $A$ is a set of arcs defined as: $((\mathrm{P}-\{M S P\}) \times \mathrm{T}) \cup((\mathrm{T} \times(\mathrm{P}-\{$ entry $\})$.

Definition 2.5 A method is a triple $(P, T, A)$, where $P$ is a set of places with three special places: entry place, ISP and return place. Each method has only one entry place and one return place, but it may contain multiple ISPs. $T$ is a set of transitions, and each transition can be associated with a set of guards. $A$ is a set of arcs defined as: ((P$\{$ return $\}) \times \mathrm{T}) \cup((\mathrm{T} \times(\mathrm{P}-\{$ entry $\})$.

\subsection{Inheritance Modeling in Agent-based G-Nets}

Although there are different views with respect to the concept of agent-oriented design [12], we consider an agent as an extension of an object, and we believe that agent-oriented design should keep most of the key features in object-oriented design. Thus, to progress from an agentbased model to an agent-oriented model, we need to incorporate some inheritance modeling capabilities. But inheritance in agent-oriented design is more complicated than in object-oriented design. Unlike an object (passive object), an agent object has mental states and reasoning mechanisms. Therefore, inheritance in agent-oriented design invokes two issues: an agent subclass may inherit an agent superclass's knowledge, goals, plans, the model of its environment and its reasoning mechanisms; on the other hand, as in the case of object-oriented design, an agent subclass may inherit all the services that an agent superclass may provide, such as private utility functions. There is existing work on agent inheritance with respect to knowledge, goals and plans [2][6]. However, we believe that since inheritance happens at the class level, an agent subclass may be initialized with an agent superclass's initial mental state, but new knowledge acquired, new plans made, and new goals generated in a individual agent object (as an instance of an agent superclass), can not be inherited by an agent object when creating an instance of an agent subclass. A superclass's reasoning mechanism can be inherited, however it is beyond the scope of this paper. For simplicity, we assume that an agent subclass always uses its own reasoning mechanisms, and thus the reasoning mechanisms in the agent superclass should be disabled in some way. This is necessary because different reasoning mechanisms may deduce different results for an agent, and to resolve this type of conflict may be timeconsuming and make an agent's reasoning mechanism inefficient. Therefore, in this paper we only consider how to initialize a subclass agent's mental state while an agent subclass is instantiated; meanwhile, we concentrate on the inheritance of services that are provided by an agent superclass, i.e., the MPUs and methods defined in the internal structure of an agent class. Before presenting our inheritance scheme, we need the following definition:

Definition 2.6 When an agent subclass $A$ is instantiated as an agent object $A O$, a unique agent identifier is generated, and all superclasses and ancestor classes of the agent subclass $A$, in addition to the agent subclass $A$ itself, are initialized. Each of those initialized classes then becomes a part of the resulting agent object $A O$. We call an initialized superclass or ancestor class of agent subclass $A$ a subagent, and the initialized agent subclass $A$ the primary subagent.

The result of initializing an agent class is to take the agent class as a template and create a concrete structure of the agent class and initialize its state variables. Since we 
represent an agent class as an agent-based G-Net, an initialized agent class is modeled by an agent-based G-Net with initialized state variables. In particular, the four tokens in the special places of an agent-based G-Net, i.e., gTkn, $p T k n, k T k n$ and $e T k n$, are set to their initial states. Since different subagents of $A O$ may have goals, plans, knowledge and environment models that conflict with those of the primary subagent of $A O$, it is desirable to resolve them in an early stage. In our case, we deal with those conflicts in the instantiation stage in the following way. All the tokens $g T k n, p T k n, k T k n$ and $e T k n$ in each subagent of $A O$ are removed from their associated special places, and these tokens are combined with the tokens gTkn, pTkn, kTkn and $e T k n$ in the primary subagent of $A O$. The resulting tokens $g T k n, p T k n, k T k n$ and $e T k n$ (newly generated by unifying those tokens for each type), are put back into the special places of the primary subagent of $A O$. Consequently, all subagents of $A O$ lose their abilities for reasoning, and only the primary subagent of $A O$ can make necessary decisions for the whole agent object. More specifically, in the Planner module (as shown in Figure 3) that belongs to a subagent, the abstract transitions make_decision, sensor and update can never be enabled because there are no tokens in the following special places: Goal, Plan and Knowledge-base. If a message tagged as internal arrives, the transition bypass may fire and a message token can directly go to a $M P U$ defined in the internal structure of the subagent if it is defined there. This is made possible by connecting the transition bypass with inhibitor arcs (denoted by dashed lines terminated with a small circle in Figure 3) from the special places Goal, Plan and Knowledge-base. So the transition bypass can only be enabled when there are no tokens in these places. In contrast to this behavior, in the Planner module of a primary subagent, tokens do exist in the special places Goal, Plan and Knowledge-base. Thus, the transition bypass will never be enabled. Instead, the transition make_decision must fire before an outgoing message is dispatched into a $M P U$ defined in the primary agent or any subagents.

To reuse the services (i.e., MPUs and methods) defined in a subagent, we need to introduce a new mechanism called Asynchronous Superclass switch Place (ASP). An $A S P$ (denoted by an ellipsis in Figure 3 ) is similar to a $M S P$, but with the difference that an $A S P$ is used to forward a message or a method call to a subagent rather than to send a message to an agent object. For the $M S P$ mechanism, the receiver could be some other agent object or the agent object itself. In the case of $M S P($ self $)$, a message token is always sent to the GSP of the primary subagent. However, for $A S P$ (super), a message token is forwarded to the $G S P$ of a subagent that is referred to by super. In the case of single inheritance, super refers to a

\footnotetext{
${ }^{1}$ The process of generating the new token values would involve actions such as conflict resolution among goals or plans, which is a topic outside the scope of our model and this paper.
}

unique superclass G-Net, however with multiple inheritance, the reference of super must be resolved by searching the class hierarchy diagram.

When a message/method is not defined in an agent subclass model, the dispatching mechanism will deposit the message token into a corresponding ASP(super). Consequently, the message token will be forwarded to the $G S P$ of a subagent, and it will be again dispatched. This process can be repeated until the root subagent is reached. In this case, if the message is still not defined at the root, an exception occurs. In this paper, we do not provide exception handling for our agent-based G-Net models, and we assume that all incoming messages have been correctly defined in the primary subagent or some other subagents.

\section{Examples of Agent-Oriented Design}

Consider an agent family in an electronic marketplace domain. Figure 4 shows the agents in a UML class hierarchy notation. A shopping agent is defined as an abstract agent that has the ability to register in a marketplace through a facilitator, which serves as a wellknown agent in the marketplace. A shopping agent cannot be instantiated as an agent object, in other words, a shopping agent cannot register itself as a shopping agent. Rather, the functionality of a shopping agent class can be inherited by an agent subclass, such as a buying agent or a selling agent. Both the buying agent object and selling agent object may reuse the functionality of a shopping agent by registering themselves as a buying agent or a selling agent through a facilitator. Furthermore, a retailer agent is an agent that can sell goods to a customer, but it also needs to buy goods from some selling agents. Thus a retailer agent class is designed as a subclass of both the buying agent class and the selling agent class. In addition, a customer agent class may be defined as a subclass of a buying agent class, and an auctioneer agent class may be defined as a subclass of a selling agent class. In this paper, we only consider four types of agent class, i.e., the shopping agent class, the buying agent class, the selling agent class and the retailer agent class. The modeling of the customer agent class and auctioneer agent class can be done in a similar way.

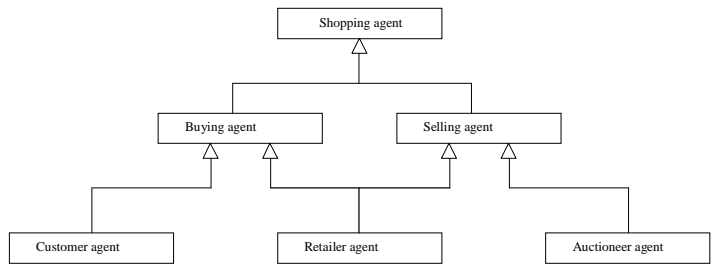

Figure 4. The class hierarchy diagram of agents in an electronic marketplace

To illustrate how to design agents by using our agent model, we use the following examples. Figure 5 (a) depicts 
a template of a contract net protocol expressed as an agent UML (AUML) sequence diagram [11] for a registrationnegotiation protocol between a shopping agent and a facilitator agent. Figure 5 (b) is a modified example of a contract net protocol adapted from [11], which depicts a template of a protocol expressed as an AUML sequence diagram for a price-negotiation protocol between a buying agent and a selling agent. Some of the notations of AUML are adapted from [11] as extensions of UML sequence diagrams for agent design. In addition, to correctly draw the sequence diagram for the protocol templates, we introduce two new notations, i.e., the end of protocol operation "•" and the iteration of communication operation “*”. Figure 5 (c) shows an example price-negotiation protocol that is instantiated from the protocol template shown in Figure 5 (b).
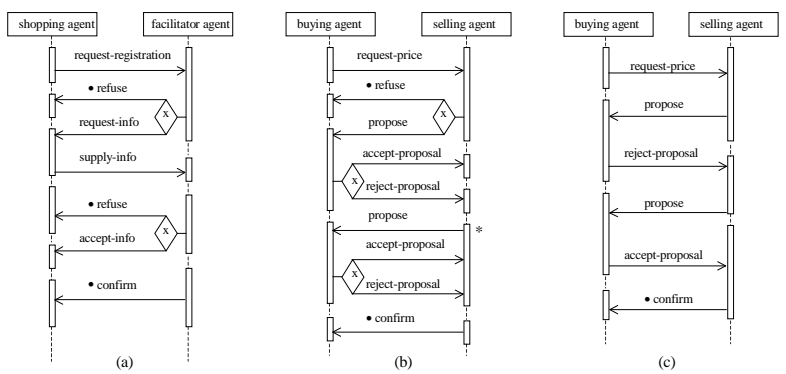

Figure 5. Contract net protocols (a) A template for the registration protocol (b) A template for the price-negotiation protocol (c) An example of the price-negotiation protocol

Consider Figure 5 (a). When a conversation based on a contract net protocol begins, the shopping agent sends a request for registration to a facilitator agent. The facilitator agent can then choose to respond to the shopping agent by refusing its registration or requesting agent information. Here the " $x$ " in the decision diamond indicates an exclusive-or decision. If the facilitator refuses the registration based on the marketplace's size, the protocol ends; otherwise, the facilitator agent waits for agent information to be supplied. If the agent information is correctly provided, the facilitator agent then still has a choice of either accepting or rejecting the registration based on the shopping agent's reputation and the marketplace's functionality. Again, if the facilitator agent refuses the registration, the protocol ends; otherwise, a confirmation message will be provided afterwards. Similarly, the price-negotiation protocol between a buying agent and a selling agent can be illustrated in Figure 5 (b). Based on the communicative acts (e.g., requestregistration, refuse, etc.) needed for the contract net protocol in Figure 5 (a), we may adopt the design template of the shopping agent shown in Figure 6. The Goal, Plan, Knowledge-base and Environment modules remain as abstract units and can be refined in a further detailed design stage. The Planner module may reuse the template shown in Figure 3. The design of the facilitator agent is similar, however it may support more protocols.
With inheritance, a buying agent class, as a subclass of a shopping agent class, may reuse MPUs/methods defined in a shopping agent class's internal structure. Similarly, based on the communicative acts (e.g., request-price, refuse, etc.) needed for the contract net protocol in Figure 5 (b), we may design the buying agent class as in Figure 7. Note that we do not define the MPUs of refuse and confirm in the internal structure of the buying agent class, for they can be inherited from the shopping agent class. A retailer agent can be designed in the same way. In addition to its own MPU/methods, a retailer agent class inherits all MPU/methods of both the buying agent class and the selling agent class.

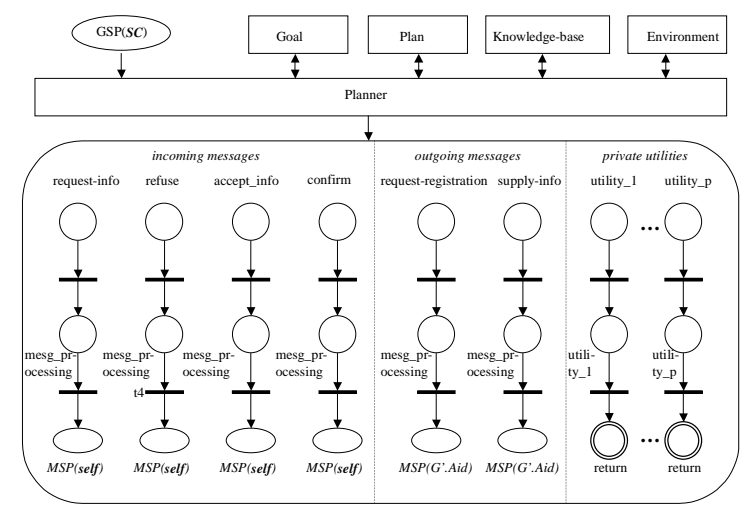

Figure 6. An agent-based G-Net model for shopping agent class

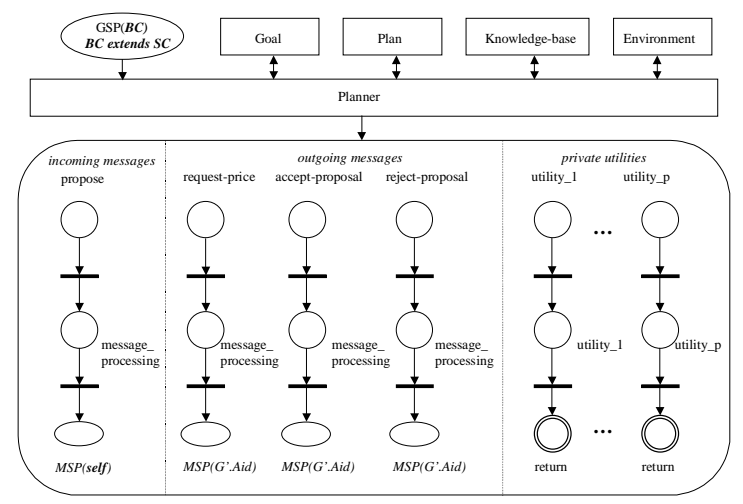

Figure 7. An agent-based G-Net model for buying agent class

Now we discuss an example to show how the reuse of MPU/methods works. Consider a buying agent object $B O$, which receives a message of request-info from a facilitator agent object $F O$. A $m T k n$ token will be deposited in the $G S P$ of the primary subagent of $B O$, i.e., the $G S P$ of the corresponding buying agent class $(B C)$. The transition external in $B C$ 's Planner module may fire, and the $m T k n$ will be moved to the place dispatch_incoming_message. Since there is no $M P U$ for request-info defined in the internal structure of $B C$, the $m T k n$ will be moved to the $A S P$ (super) place. Since super here refers to a unique superclass - the shopping agent class $(S C)$ - the $m T k n$ will 
be transferred to the GSP of SC. Now the $m T k n$ can be correctly dispatched to the $M P U$ for request-info. After the message is processed, $M S P($ self $)$ changes the tag of the $m T k n$ from external to internal, and sends the processed $m T k n$ token back into the $G S P$ of $B C$. Note that $M S P($ self $)$ always sends a $m T k n$ back to the GSP of the primary subagent. Upon the arrival of this message token, the transition internal in the Planner module of $B C$ may fire, and the mTkn token will be moved to the place check_primary. Since $B C$ corresponds to the primary subagent of $B O$, there are tokens in the special places Goal, Plan, Knowledge-base and Environment. Therefore the abstract transition make_decision may fire, and any necessary actions are executed in place next_action. Then the current conversation is either ignored or continued based on the decision made in the abstract transition make_decision. If the current conversation is ignored, the goals, plans and knowledge-base are updated as needed; otherwise, in addition to the updating of goals, plans and knowledge-base, a newly constructed $m T k n$ with a tag of external is deposited into place dispatch_outgoing_message. The new $m T k n$ token has the message name supplyinfo, following the protocol defined in Figure 5 (a). Again, there is no $M P U$ for supply-info defined in $B C$, so the new $m T k n$ token will be dispatched into the GSP of $S C$. Upon the arrival of the $m T k n$ in the GSP of $S C$, the transition external in the Planner module of $S C$ may fire. However at this time, $S C$ does not correspond to the primary subagent of $B O$, so all the tokens in the special places of Goal, Plan, Knowledge-base have been removed. Therefore, the transition bypass is enabled. When the transition bypass fires, the $m T k n$ token will be directly deposited into the place dispatch_outgoing_message, and now the $m T k n$ token can be correctly dispatched into the $M P U$ for supply-info defined in SC. After the message is processed, the $m T k n$ token will be transferred to the GSP of the receiver mTkn.body.receiver, and in this case, it is a facilitator agent object.

For the reuse of private utility functions defined in a superclass, the situation is the same as in the case of object-oriented design. Examples concerning reuse of private utility functions and different forms of inheritance, such as augment inheritance and restrictive inheritance, can be found in [9].

\section{Conclusions and Future Work}

One of the most rapidly growing areas of interest for distributed computing is that of distributed agent systems. In this paper, we introduced a framework of agent models with an example of agent family in electronic commerce. Using this framework, shopping agent, selling agent, buying agent and retailer agent can be modeled as intelligent agents with the characteristics of being autonomous, reactive and internally-motivated. Examples of a registration-negotiation protocol between shopping agents and facilitator agents, and a price-negotiation protocol between a shopping agents and buying agents were used to illustrate our basic idea.

For our future work, we will consider the refinement of the Goal, Plan, Knowledge-base and Environment modules. The abstract transitions defined in the Planner module, i.e., make_decision, sensor and update, will be refined into correct sub-nets too. We will also look into issue like deadlock avoidance and state exploration problems in the agent-oriented design and verification processes.

\section{References}

[1] S. Green, L. Hurst, B. Nangle, P. Cunningham, F. Somers, R. Evans, "Software Agents: A Review," Technical report TCD-CS-1997-06, Trinity College Dublin, May 1997.

[2] David Kinny, Michael P. Georgeff, "Modeling and Design of Multi-Agent Systems," Proceedings of the 4th Int'l Workshop on Agent Theories, Architectures, and Language (ATAL-97), 1997, pp. 1-20.

[3] T. J. Rogers, R. Ross, V. S. Subrahmanian, "IMPACT: A System for Building Agent Applications," Journal of Intelligent Information Systems, 14(2-3): 95-113, 2000.

[4] Carlos Argel Iglesias, Mercedes Garrijo, José CentenoGonzález, "A Survey of Agent-Oriented Methodologies," Proceedings of the Fifth International Workshop on Agent Theories, Architectures, and Language (ATAL-98), 1998, pp. 317-330.

[5] D. Kinny, M. Georgeff, and A. Rao, "A Methodology and Modeling Technique for Systems of BDI Agents," Tech. Rep. 58, Australian Artificial Intelligence Institute, Melbourne, Australia, Jan. 1996.

[6] Lobel Crnogorac, Anand S. Rao, Kotagiri Ramamohanarao, "Analysis of Inheritance Mechanisms in Agent-Oriented Programming," IJCAI (1) 1997: 647-654.

[7] T. Murata, "Petri Nets: Properties, Analysis and Applications," Proceedings of the IEEE, 77(4): 541-580, April 1989.

[8] A. Perkusich and J. de Figueiredo, "G-Nets: A Petri Net Based Approach for Logical and Timing Analysis of Complex Software Systems," Journal of Systems and Software, 39(1): 39-59, 1997.

[9] Haiping $\mathrm{Xu}$ and Sol Shatz, "Extending G-Nets to Support Inheritance Modeling in Concurrent Object-Oriented Design," IEEE Int'l Conf. on Systems, Man, and Cybernetics, Nashville, Tenn., Oct. 2000, pp. 3128-3133.

[10] Tim Finin, Yannis Labrou, and James Mayfield, "KQML as an agent communication language," in Jeff Bradshaw (Ed.), Software Agents, MIT Press, Cambridge, 1997.

[11] James Odell, H. Van Dyke Parunak, Bernhard Bauer, "Representing Agent Interaction Protocols in UML," ICSE 2000 Workshop on Agent-Oriented Software Engineering (AOSE-2000), June 10, 2000, Limerick, Ireland.

[12] M. Wooldridge, N. R. Jennings, and D. Kinny, "The Gaia Methodology for Agent-Oriented Analysis and Design," International Journal of Autonomous Agents and MultiAgent Systems, 3(3): 285-312, 2000. 